\title{
Facile Method to Prepare Superhydrophobic and Water Repellent Cellulosic Paper
}

\author{
Ioannis Karapanagiotis, ${ }^{1,2}$ Diana Grosu, ${ }^{1}$ Dimitra Aslanidou, ${ }^{1}$ and Katerina E. Aifantis ${ }^{2,3}$ \\ ${ }^{1}$ Department of Management and Conservation of Ecclesiastical Cultural Heritage Objects, \\ University Ecclesiastical Academy of Thessaloniki, 54250 Thessaloniki, Greece \\ ${ }^{2}$ Lab of Mechanics and Materials, Aristotle University of Thessaloniki, 54124 Thessaloniki, Greece \\ ${ }^{3}$ Department of Civil Engineering, University of Arizona, Tucson, AZ 85781, USA \\ Correspondence should be addressed to Ioannis Karapanagiotis; y.karapanagiotis@aeath.gr
}

Received 3 December 2014; Revised 26 January 2015; Accepted 26 January 2015

Academic Editor: Enrico Bergamaschi

Copyright (C) 2015 Ioannis Karapanagiotis et al. This is an open access article distributed under the Creative Commons Attribution License, which permits unrestricted use, distribution, and reproduction in any medium, provided the original work is properly cited.

\begin{abstract}
Silica nanoparticles $(7 \mathrm{~nm})$ were dispersed in solutions of a silane/siloxane mixture. The dispersions were applied, by brush, on four types of paper: (i) modern, unprinted (blank) paper, (ii) modern paper where a text was printed using a common laser jet printer, (iii) a handmade paper sheet detached from an old book, and (iv) Japanese tissue paper. It is shown that superhydrophobicity and water repellency were achieved on the surface of the deposited films, when high particle concentrations were used $(\geq 1 \% \mathrm{w} / \mathrm{v})$, corresponding to high static $\left(\theta_{S} \approx 162^{\circ}\right)$ and low tilt $\left(\theta_{t}<3^{\circ}\right)$ contact angles. To interpret these results, scanning electron microscopy (SEM) was employed to observe the surface morphologies of the siloxane-nanoparticle films. Static contact angles, measured on surfaces that were prepared from dilute dispersions (particle concentration $<1 \% \mathrm{w} / \mathrm{v}$ ), increased with particle concentration and attained a maximum value $\left(162^{\circ}\right)$ which corresponds to superhydrophobicity. Increasing further the particle concentration did not have any effect on $\theta_{S}$. Colourimetric measurements showed that the superhydrophobic films had negligible effects on the aesthetic appearance of the treated papers. Furthermore, it is shown that the superhydrophobic character of the siloxane-nanoparticle films was stable over a wide range of $\mathrm{pH}$.
\end{abstract}

\section{Introduction}

Superhydrophobic surfaces have attracted considerable attention in the last two decades because of their various potential applications relevant to water repellency, self-cleaning, friction reduction, and antifouling [1]. Although superhydrophobicity has been known since the 1940s [2], the subject has been recently revisited, as technological developments provide the capability to study, control, and manipulate materials at the micro- and nanoscales. In 1997, it became clear that superhydrophobicity in nature occurs on textured surfaces with hierarchical micrometer and nanometer sized structures in connection to hydrophobic surface components, for example, the surface of the lotus leaf [3]. Since then, significant research has focused on the fabrication of superhydrophobic surfaces, mimicking the famous example of the surface of the lotus leaf.
Paper is an inherent hydrophilic material because of the hydroxyl groups contained in cellulose. The inherent hydrophilic nature of cellulose poses obvious limitations in the use of paper, when hydrophobicity is highly demanded. For example, water/moisture resistance of paper is important for the packaging industry [4]. Conventional, hydrophobic barrier coatings are sometimes not enough to meet the requirements necessary for packaging food and drink. Superhydrophobic, water repellent coatings can provide enhanced protection against water and moisture. Another potential application of water repellent films on paper is related to the preservation of documents and books, which is aided by the unique antisticking and self-cleaning property. Finally, developing strategies to control the interaction of paper with water is important in the development of inexpensive microfluidic paper-based devices used for different purposes 
such as cell based biosensors and biochemical analytical systems [5].

The modification of the chemical composition of the surface is necessary to change the wettability of the waterloving cellulosic paper (i.e., the interaction between paper and water) to being hydrophobic. Further enhancement of hydrophobicity to achieve superhydrophobicity implies that the surface structure should be modified appropriately to induce micro- and nanoscale roughness [3]. Within this spirit, some methods were devised and suggested to produce superhydrophobic paper including chemical grafting where the hydroxyl groups are reacted to provide cellulose derivatives $[6,7]$, solution immersion and deposition $[8,9]$, deposition of polymer-nanoparticle composites followed by fluorination surface treatment [10], electrospray deposition [11], expanding supercritical fluids [12], and, quite often, nanoparticle deposition [13-19].

In the following, we describe an extremely simple (facile) method to induce superhydrophobicity on paper: silica $\left(\mathrm{SiO}_{2}\right)$ nanoparticles $(7 \mathrm{~nm})$ are dispersed in a silane/siloxane solution, and then the dispersions are used to coat four types of paper by brush. The resulting coatings have superhydrophobic properties, provided that an appropriate concentration of nanoparticles was used in the dispersions. The method presented herein has some important advantages: (i) it is an easy, one-step method implying that the two key parameters of superhydrophobicity, surface energy reduction and roughness creation, are accomplished simultaneously and not in sequence, as what happens in two-step or multiplestep methods. (ii) It is a low cost method as it does not include the use of any sophisticated instrumentation or expensive materials. Furthermore, the method is very friendly to the end user, as the siloxane-nanoparticle dispersion is applied on paper by brush, thus implying that no specialised staff is necessary. (iii) The method can be easily used for the treatment of large paper surfaces. (iv) The deposited films are transparent, as they do not affect practically the colouring appearance of the paper substrates. (v) Finally, it is stressed that the obtained superhydrophobicity is clearly accompanied by water repellency, as evidenced by the extremely small tilt contact angles measured on the surfaces of the produced films.

\section{Experimental Section}

Silres BS-290, a solventless silicone concentrate that is based on silane/siloxane (Wacker), was dissolved in white spirit (SHELLSOL H, Shell Chemicals) to prepare a stock solution of $7 \%$ wt. Silica $\left(\mathrm{SiO}_{2}\right)$ particles (fumed powder, Aldrich) with a $7 \mathrm{~nm}$ mean diameter were dispersed in the silane/siloxane solution in various concentrations. Dispersions were stirred for $20 \mathrm{~min}$ and applied on paper by brush. Films were deposited with four brush strokes and were left to cure/polymerise, according to the scheme of Figure 1, for more than $24 \mathrm{~h}$ at room temperature [20], resulting in solid networks of siloxane, enriched with $\mathrm{SiO}_{2}$ nanoparticles, that is, siloxane-particle composite films. For comparison, pure Silres BS-290 solution (without particles) was deposited on paper.
Polycondensation scheme

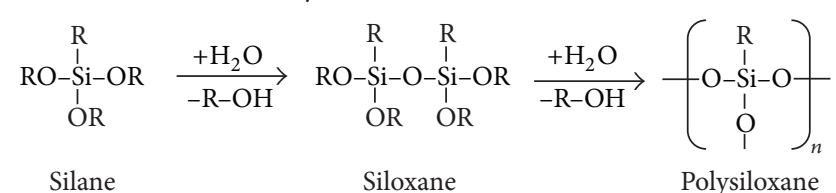

FIGURE 1: Scheme of the curing/polymerization process of silanes/ siloxanes in room condition. A siloxane network (polysiloxane) is produced under the influence of air humidity.

The aforementioned procedure was used to treat four types of cellulosic paper: common, standard A4 size paper (Asia Pacific Resources International Limited, April) which is provided for printing purposes, was purchased from the local market and used as received (modern blank paper). On another sheet of blank paper, a text was printed using a Hewlett Packard P3005 laser jet and used in the study (modern printed paper). A handmade paper sheet, detached from an old book (early 20th century) which was written in Greek, was the third paper substrate studied and labelled "old paper." Finally, Japanese tissue paper, which is used in book and paper conservation and restoration, was included and treated as described above.

Contact angles were measured using an optical tensiometer apparatus (Attension Theta) and distilled water. The reported contact angles are averages of five measurements, which were carried out on different areas of each sample. The variations are given as error bars. For the measurements of the tilt angles, the tilt rate was adjusted to $1^{\circ} / \mathrm{s}$. The volume of the droplets was $8 \mu \mathrm{L}$.

Scanning electron microscopy (SEM; JEOL, JSM-6510) was employed to study the surface structures of the samples, which were coated with a thin layer of carbon. Furthermore, SEM was used to estimate the thickness (or the amount) of the deposited films which was around $10 \mu \mathrm{m}$ according to SEM cross-sectional imaging. It is noted that this thickness result was achieved for films deposited by brush on glass, as the films applied on paper may penetrate the paper fibres.

Colourimetric measurements were carried out with a Miniscan EZ (HunterLab) instrument and the results were evaluated using the $L^{*}, a^{*}$, and $b^{*}$, coordinates of the CIE 1976 scale.

In order to examine the effect of the $\mathrm{pH}$ of the droplet on the wettability of the films, solutions with a wide range of $\mathrm{pH}(0.38-13.79)$ were prepared using hydrochloric acid $(\mathrm{HCl})$ and sodium hydroxide $(\mathrm{NaOH})$. Droplets of these solutions were placed on siloxane-particle composite films, which had been deposited on blank paper, and contact angles were measured. For comparison the same measurements were carried out on pure siloxane films (no particles).

In another brief experiment, a piece of modern blank paper sample was coated using an airbrush system (Paasche Airbrush) with a nozzle of $733 \mu \mathrm{m}$ in diameter. According to this process a siloxane-particle dispersion was deposited on paper by spray and the wettability of the produced surface was compared with the results obtained using the standard deposition process with brush. 


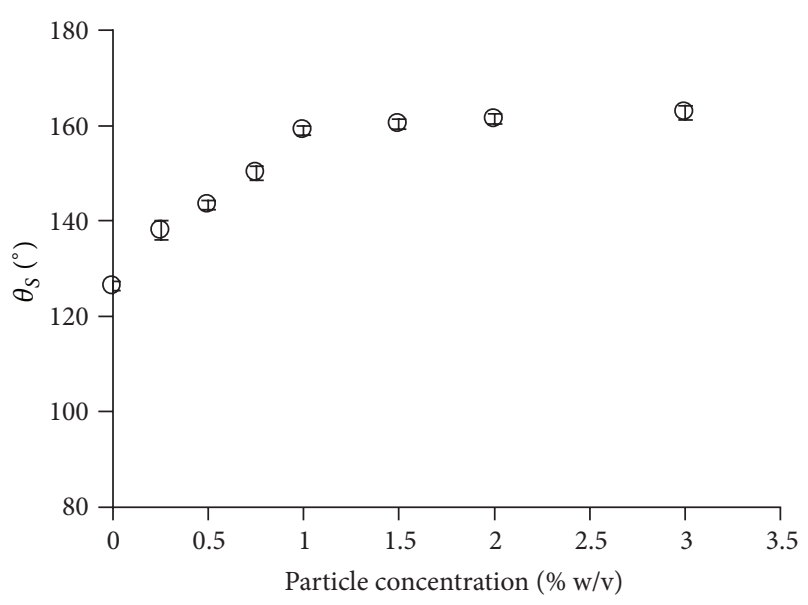

Figure 2: Static contact angle $\left(\theta_{S}\right)$ versus the $\mathrm{SiO}_{2}$ particle concentration dispersed in the siloxane solution. Films were deposited on modern blank paper. Error bars are included.

Finally, solution of pure siloxane Silres BS-290 (no particles) was spin-coated onto glass slides. The produced smooth surface was used to estimate the Young contact angle of the siloxane material, which was $97^{\circ}$, thus suggesting that Silres BS-290 is an inherently hydrophobic material. This was the only use of the spin coating technique.

\section{Results and Discussion}

Figure 2 shows the static contact angle $\left(\theta_{S}\right)$ as a function of particle concentration for films deposited on modern blank paper. The contact angle of the droplet placed on pure (no particles) siloxane film was $126^{\circ}$ and increased up to around $162^{\circ}$ for dispersions of high particle concentration $(\geq 1 \% \mathrm{w} / \mathrm{v})$. In particular, Figure 2 shows that $\theta_{S}$ increases initially with particle concentration and then it is stabilized to a constant value $\left(\sim 162^{\circ}\right)$ obtained at, roughly, $1 \% \mathrm{w} / \mathrm{v}$ particle concentration. Further increase in particle concentration does not have any significant effect on $\theta_{S}$ which is constant corresponding to the plateau of the curve in Figure 2. Superhydrophobicity $\left(\theta_{S}=150^{\circ}\right)$ is achieved for particle concentration of $\sim 0.75 \% \mathrm{w} / \mathrm{v}$. The superhydrophobic character of the surface is enhanced when the concentration increases up to $1 \% \mathrm{w} / \mathrm{v}$ where it becomes saturated as it does not change by adding more nanoparticles.

The results of Figure 2 are in agreement with previously published studies which described the effect of nanoparticles on the surface of polymer-nanoparticle composites [21-24]. Nanoparticles form microscale clusters which enhance the micrometer/nanometer scale roughness of the surface. As described in the Introduction, roughness is a key parameter to achieve superhydrophobicity. Figures 3(a), 3(c), 3(e), and $3(\mathrm{~g})$ show the surfaces of pure hydrophobic siloxane films deposited on blank, printed, old, and Japanese papers, respectively. In each of the four aforementioned SEM images, a continuous film is shown. Some surface anomalies that can be seen in the SEM images originated either from the roughness of the underlying paper substrate or the film deposition process (brush). Figures 3(b), 3(d), 3(f), and 3(h) show the surfaces of the composite siloxane-particle films which were prepared using $2 \% \mathrm{w} / \mathrm{v} \mathrm{SiO}_{2}$ nanoparticles and were deposited on the four aforementioned types of paper. Protruded particlesiloxane aggregates are formed which enhance roughness at the micrometer scale. The randomly distributed aggregates consist of further nanostructures implying that a two-lengthscale hierarchical structure is formed on the surface, which is responsible for the observed superhydrophobicity [3].

Figures 3(i) and 3(j) show SEM images of higher magnifications focusing on specific areas of Figures 3(g) (hydrophobic surface) and 3(h) (superhydrophobic surface), as indicated by the dotted lines. In particular, the surface structure of an area which exists between the protruded aggregates (dotted line in Figure 3(h)) is revealed in Figure 3(j). The image in Figure 3(i) is provided for comparative purposes. The two high magnification images reveal the cellulosic fibres of the treated Japanese paper. Furthermore, they show that the areas which exist between the protruded aggregates in superhydrophobic surfaces (Figure 3(j)) do not exhibit any special structure. On the contrary, the surface structure in Figure $3(\mathrm{j})$ is very similar to the surface morphology of pure (hydrophobic) siloxane, shown in Figure 3(i). Consequently, the morphological differences of the hydrophobic and superhydrophobic surfaces are attributed exclusively on the aggregates that are formed by the use of nanoparticles, as discussed above.

Photographs of typical water droplets placed on hydrophobic and superhydrophobic surfaces are included in Figures 3(a) and 3(b), respectively. The diameters of the contact areas of these droplets were measured and found to be 1.8 (Figure 3(a)) and $1.0 \mathrm{~mm}$ (Figure 3(b)). These values are lower than the capillary length $\kappa^{-1}$ which is the threshold above which gravity cannot be neglected compared to capillary forces:

$$
\kappa^{-1}=\sqrt{\frac{\gamma}{\rho g}}
$$

where $\gamma$ and $\rho$ are the surface tension and density of water, respectively, and $g$ is the acceleration due to gravity. At room temperature (1) gives $\kappa^{-1}=2.7 \mathrm{~mm}$. This length is clearly longer than the contact diameters of the typical water droplets shown in Figures 3(a) and 3(b). Consequently, gravitational effects on the measured $\theta_{S}$ which are reported in the present investigation can be considered as negligible.

Contact angle measurements on the four types of paper which were covered by pure siloxane (no particles) and siloxane-particle composite films are described in Table 1. Interestingly, $\theta_{S}$ reported in Table 1 for pure (no particles) siloxanes are $\left(>120^{\circ}\right)$ larger than the $97^{\circ}$ angle measured on pure siloxane spin coated on glass, as described in the Experimental Section. The surface of the film deposited on glass by spin coating is extremely smooth. However, as shown in Figures 3(a), 3(c), 3(e), and 3(g), the surfaces of the films deposited by brush on paper sheets exhibited roughness that resulted in elevated $\theta_{S}\left(>120^{\circ}\right)$ according to the values reported in Table 1. 
TABLE 1: Static $\left(\theta_{S}\right)$ and tilt $\left(\theta_{t}\right)$ contact angles measured on four paper surfaces, which were covered by pure siloxane and siloxane-particle films, corresponding to 0 and $2 \% \mathrm{w} / \mathrm{v}$ particle concentration, respectively.

\begin{tabular}{lccccc}
\hline \multirow{2}{*}{ Contact angle $\left(^{\circ}\right)$} & \multirow{2}{*}{ Particle concentration $(\mathrm{w} / \mathrm{v})$} & \multicolumn{3}{c}{ Paper substrate } \\
& & Blank paper & Printed paper & Old paper & Japanese paper \\
\hline \multirow{2}{*}{$\theta_{S}$} & 0 & $126.4 \pm 0.3$ & $121.0 \pm 0.9$ & $126.0 \pm 0.6$ & $122.2 \pm 0.5$ \\
& $2 \%$ & $161.3 \pm 0.8$ & $164.7 \pm 0.7$ & $163.2 \pm 1.1$ & $162.0 \pm 1.2$ \\
\hline \multirow{2}{*}{$\theta_{t}$} & 0 & $10.8 \pm 0.1$ & $11.0 \pm 0.3$ & $10.9 \pm 0.7$ & $10.8 \pm 0.6$ \\
& $2 \%$ & $2.4 \pm 0.3$ & $1.8 \pm 0.3$ & $2.1 \pm 0.2$ & $2.3 \pm 0.4$ \\
\hline
\end{tabular}

Both static $\left(\theta_{S}\right)$ and tilt $\left(\theta_{t}\right)$ contact angles are included in Table 1. The former $\left(\theta_{S}\right)$ is used to describe the hydrophobic/hydrophilic character of a surface which is assessed by placing a resting water drop on a horizontal surface. The latter $\left(\theta_{t}\right)$ is related to the water repellency/adhesion. The terms "hydrophobicity/hydrophilicity" and "water repellency/adhesion" should be carefully used. For example, the surface of the red rose petal exhibits superhydrophobicity as it corresponds to $\theta_{S}>150^{\circ}$, and yet at the same time, a droplet cannot roll off even when the petal is turned upside down (i.e., $\theta_{t}$ tends to $180^{\circ}$ ), thus implying high droplet adhesion [25]. On the other hand, the surface of the lotus leaf exhibits superhydrophobic and water repellent properties, as $\theta_{S}>$ $150^{\circ}$ and $\theta_{t}<5^{\circ}[26]$.

The results of Table 1 show that lotus leaf-like wettability was induced by the nanoparticles. When pure siloxane was deposited on the paper surfaces, relatively low $\left(<130^{\circ}\right)$ and high $\left(>10^{\circ}\right) \theta_{S}$ and $\theta_{t}$ were measured, respectively. However, the wettability of the siloxane-particle films follows the properties of the lotus leaf, as contact angles are $\theta_{S}>160^{\circ}$ and $\theta_{t}<3^{\circ}$. As silica is a hydrophilic material, it is safe to conclude that the $\mathrm{SiO}_{2}$ nanoparticles only contribute to surface roughness, while it is the siloxane that is in the topmost layer all over the surface $[21,24,27]$. That is, films prepared with and without $\mathrm{SiO}_{2}$ particles have similar surface chemistry, and it is their surface roughness (Figure 3 ) that is responsible for the difference in wettability observed.

According to the results of Table 1, the surfaces of the siloxane-particle films exhibit nonsticking properties, thus implying that they can be described by the Cassie-Baxter equation which correlates the elevated apparent static contact angle $\left(\theta^{*}\right)$ and the contact angle $\left(\theta_{Y}\right)$ measured on a smooth surface [2]:

$$
\cos \theta^{*}=-1+f_{s}\left(\cos \theta_{Y}+1\right)
$$

where $f_{s}$ is the surface porosity factor, that is, the fraction of the superhydrophobic surface which is in direct contact with water.

Using (2), the $f_{s}$ factors of the superhydrophobic composite films can be calculated, considering that $\theta_{Y}$ is $97^{\circ}$, as measured for the smooth spin coated siloxane, and $\theta^{*}$ corresponds to the $\theta_{S}$ angles reported in Table 1 for the siloxane-particle superhydrophobic surfaces. For the lower $\left(161.3^{\circ}\right)$ and higher $\left(164.7^{\circ}\right)$ values of $\theta_{S}$ reported in Table 1 we calculate $f_{s}=0.06$ and $f_{s}=0.04$, respectively. These values are in agreement with corresponding measurements reported in previous studies. For example, Basu and Kumar studied the wettability of composite coatings which were fabricated by incorporating hydrophobically modified silica nanoparticles $(20 \mathrm{~nm})$ in polytetrafluoroethylene (PTFE) [23]. Using the Cassie-Baxter equation, they calculated that, for the superhydrophobic composite, $f_{s}=0.052$ [23]. Schutzius et al. studied the wettability of polysilsesquioxane-silica composite coatings prepared by spray casting nanoparticle dispersions. They described the wettability of these surfaces using the Cassie-Baxter equation and reported that $f_{s}=0.04$ [24].

The force $(F)$ needed to start a drop moving over a solid inclined can be calculated using the following simple formula $[28,29]$ :

$$
F=m g \sin \theta_{t}
$$

where $m$ is the mass of the droplet. Using the $\theta_{t}$ values reported in Table 1, we calculate that it takes 4.5 to 6.1 times as much force to move a droplet on pure siloxane than on siloxane-particle composite film. The lower (4.5) and upper (6.1) limits correspond to modern blank and printed paper, respectively. Consequently, water drops can roll off the composite siloxane-particle surface (water repellency) easier compared to the pure siloxane "sticky" surface (water adhesion).

The enhanced droplet mobility on siloxane-particle surfaces induced the self/easy-cleaning property, which is demonstrated in Figure 4. The surface of a superhydrophobic siloxane-particle film on paper was intentionally contaminated with red pigment particles which were easily removed by water without leaving any stain on the treated paper (Figure 4(a)). The same process was repeated on pure, hydrophobic siloxane film. The removal of the red pigment by water was unsuccessful, as it resulted in red stains on the surface of the treated paper (Figure 4(b)).

The angles reported in Table 1 were measured for films deposited on paper samples with the four-brushing hand application procedure. We report that superhydrophobicity and water repellency were achieved for siloxane-particle films deposited by spray. Contact angles measured for siloxaneparticle films, which were prepared using $2 \% \mathrm{w} / \mathrm{v} \mathrm{SiO}_{2}$ nanoparticles and sprayed on blank paper, were $\theta_{S}=161.5^{\circ} \pm$ 1.0 and $\theta_{t}=2.8^{\circ} \pm 0.2$. These results are extremely close to the angles reported in Table 1, implying that the brushing hand application procedure can be replaced by a more viable technological process, such as spray deposition.

A protective film designed for the paper industry should be transparent, implying that the film should not affect the aesthetic appearance of the underlying paper substrate. 


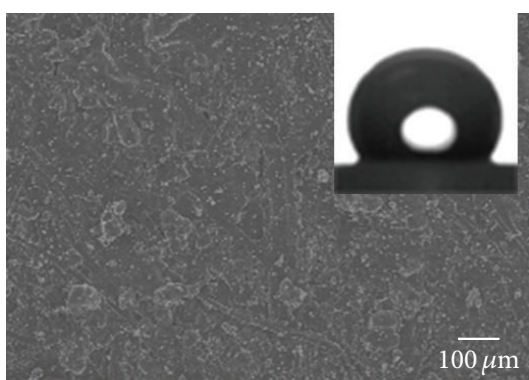

(a)

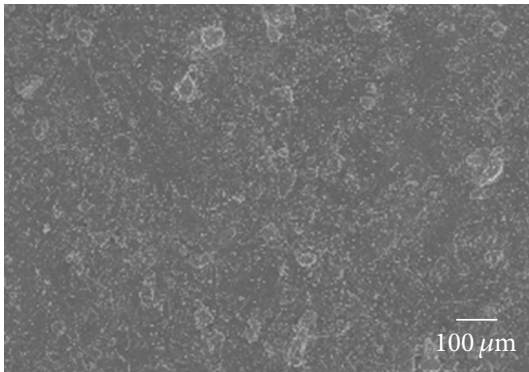

(c)

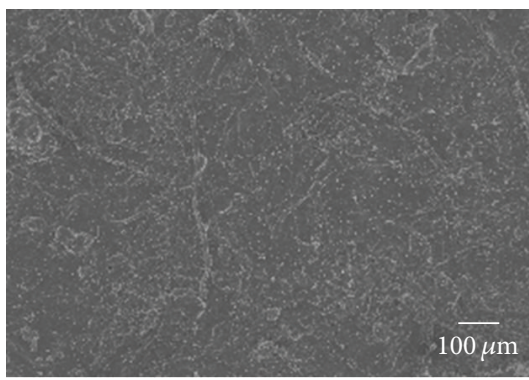

(e)

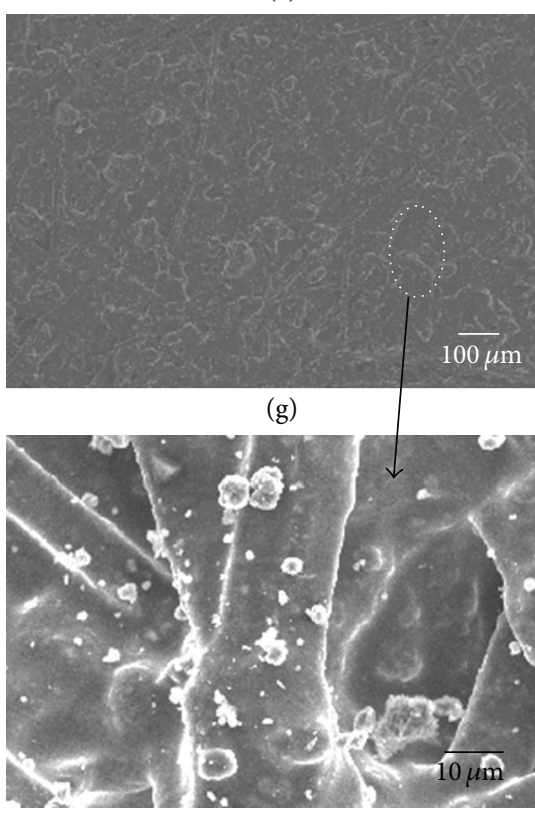

(i)

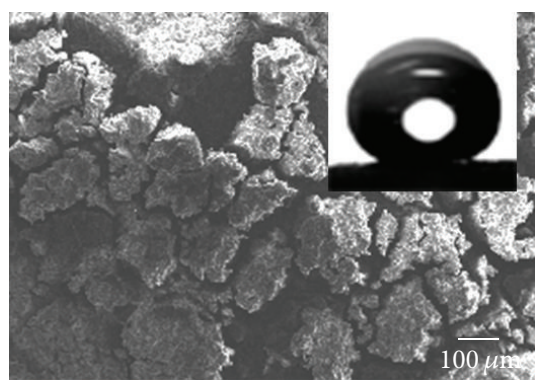

(b)

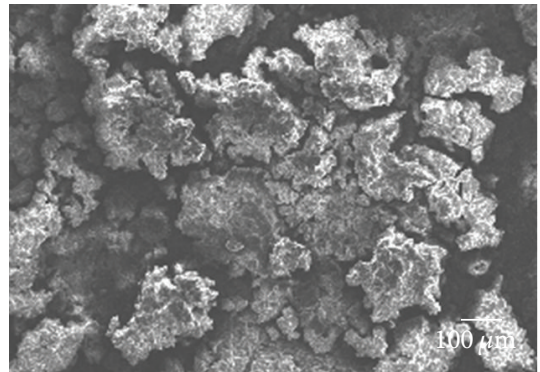

(d)

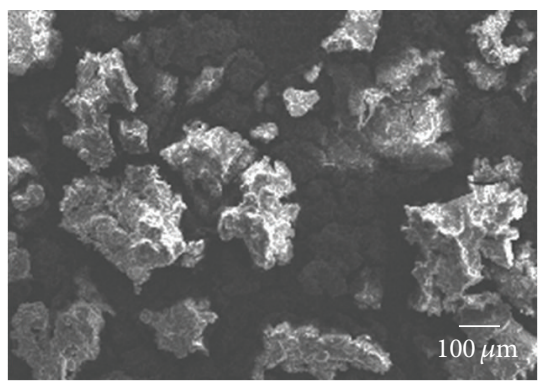

(f)

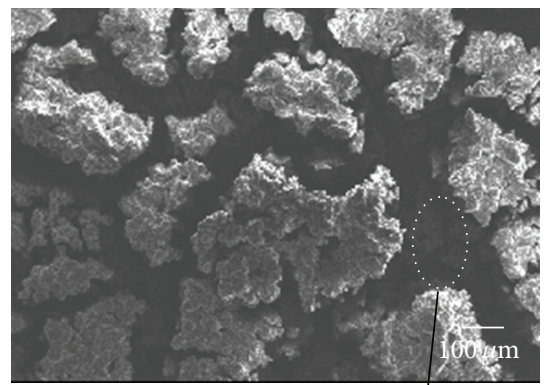

(h)

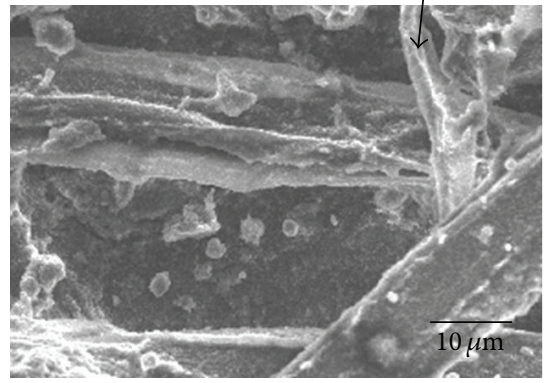

(j)

Figure 3: SEM images of (a, c, e, and g) hydrophobic pure siloxane and (b, d, f, and h) superhydrophobic siloxane-particle (2\% w/v) films deposited on ( $\mathrm{a}, \mathrm{b})$ modern blank, (c, d) modern printed, (e, f) old, and (g, h) Japanese paper. Specific areas of Figures (g) and (h) are revealed in higher magnification SEM images in Figures (i) and (j), respectively. The image in (j) corresponds to an area that exists between the protruded aggregates, indicated by the dotted line in (h). The area shown in (i) was randomly selected and is provided for comparative purposes. Photographs of water drops are shown in (a) and (b). 

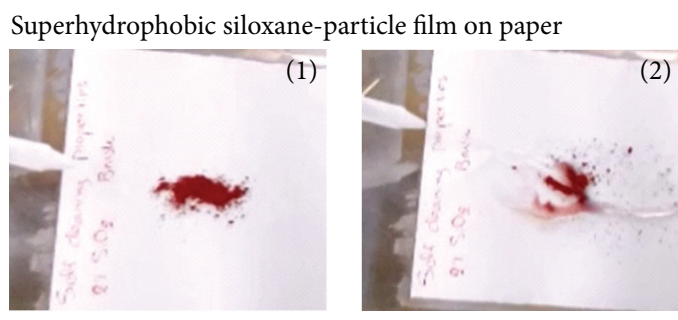

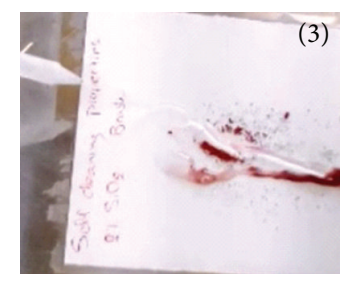

(a)

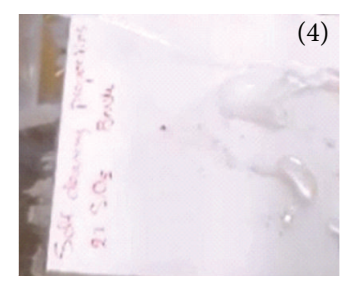

4)

(2)
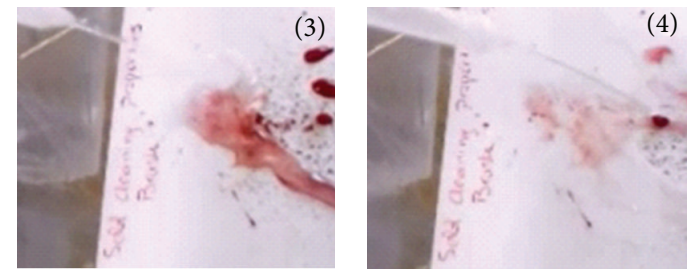

(b)

FIGURE 4: (a) The easy/self-cleaning process on a water-repellent surface of a siloxane-particle film on paper is demonstrated in the successive snapshots. The film surface was intentionally contaminated with reddish particles which were removed by water. (b) The same process was repeated on a hydrophobic siloxane film on paper. The treated paper was stained by the red particles, upon water flow.

Hence, colourimetric measurements were carried out on paper samples, which were (i) untreated and used as received, (ii) coated by pure siloxane (hydrophobic) films, and (iii) coated by siloxane-particle (superhydrophobic) films. The global colour differences $\left(\Delta E^{*}\right)$ of the specimens due to film application were calculated according to [30]

$$
\Delta E^{*}=\sqrt{\Delta L^{* 2}+\Delta a^{* 2}+\Delta b^{* 2}},
$$

where $L^{*}, a^{*}$, and $b^{*}$ are the brightness ( 0 for black, 100 for white), the red-green component (positive for red and negative for green), and the yellow-blue component (positive for yellow and negative for blue), respectively. The results are described in Table 2. Films, consisted of either pure siloxane or siloxane enriched with nanoparticles, do not have any visible effect on the aesthetic appearance of modern blank and printed paper. Colour changes recorded in these samples correspond to $\Delta E^{*}<3$ and are therefore not visible by naked eye [30]. Similarly, the appearance of old paper remained practically unaffected by the application of the superhydrophobic siloxane-particle film. It is noteworthy that white silica nanoparticles had a positive effect on the appearance of old paper as they did reduce $\Delta E^{*}(=1.35)$ compared to the global colour difference measured for old paper treated with pure siloxane (=3.24). The same effect is observed for the modern printed paper. The highest values of $\Delta E^{*}$ were recorded for the treated Japanese paper, where $\Delta E^{*}$ correspond to 4.34 and 4.80 for siloxane and siloxane-particle films, respectively. These colour changes, however, are hardly visible by naked eye as demonstrated in the photographs of Figure 5.

Based on the results of Table 2 and Figure 5, it was concluded that the use of $\mathrm{SiO}_{2}$ nanoparticles does not have any noticeable negative impact on the aesthetic appearance of the studied papers. On the contrary, for modern printed and old paper, nanoparticles tend to reduce the negative effect raised by the siloxane. Consequently, the wettability transition from hydrophobicity, obtained with films of pure siloxane, to superhydrophobicity, induced by using films of siloxane enriched with $\mathrm{SiO}_{2}$ nanoparticles, is not accompanied by a colourimetric/aesthetic penalty.

Finally, it is interesting to note that the superhydrophobic character of the siloxane-particle films remained unaffected for a wide $\mathrm{pH}$ range of water droplets. Figure 6 shows $\theta_{S}$ versus the $\mathrm{pH}$ of droplets that were placed on a siloxaneparticle film, deposited by brush on modern blank paper. It is shown that $\theta_{S}$ is stable over a wide range of $\mathrm{pH}$, thus suggesting that the superhydrophobic character of the film is stable for acidic and basic aqueous droplets. This stability originates from the corresponding stability of the siloxane (Silres BS-290) material as demonstrated by the results of Figure 6, which shows also that $\theta_{S}$ measured on pure siloxane (no particles) is constant for a wide range of $\mathrm{pH}$. A decrease of $\theta_{S}$ for both pure siloxane and siloxane-particle films is observed only for extreme basic $(\mathrm{pH}=13.79)$ conditions, as shown in Figure 6.

\section{Conclusions}

A low-cost, simple (facile) method was presented that can be used to induce superhydrophobicity and water repellency on paper on a routine basis: silica $\left(\mathrm{SiO}_{2}\right)$ nanoparticles $(7 \mathrm{~nm})$ were dispersed in a silane/siloxane solution. After mechanical agitation, the dispersion was applied on paper by brush. The surface of the deposited film exhibited superhydrophobic $\left(\theta_{S}>160^{\circ}\right)$, water repellent $\left(\theta_{t}<3^{\circ}\right)$, and self-cleaning properties, provided that the particle concentration added in the dispersion was $\geq 1 \% \mathrm{w} / \mathrm{v}$. SEM images showed that nanoparticles resulted in the formation of surface structures 


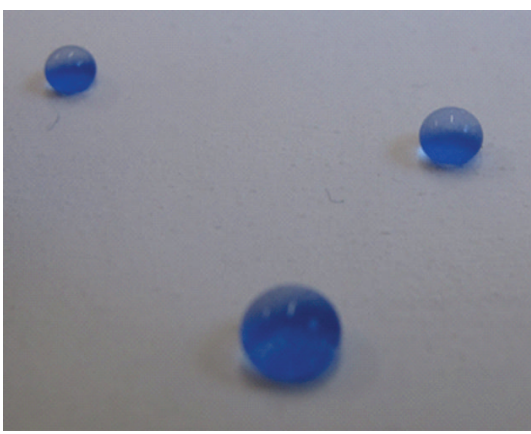

(a)

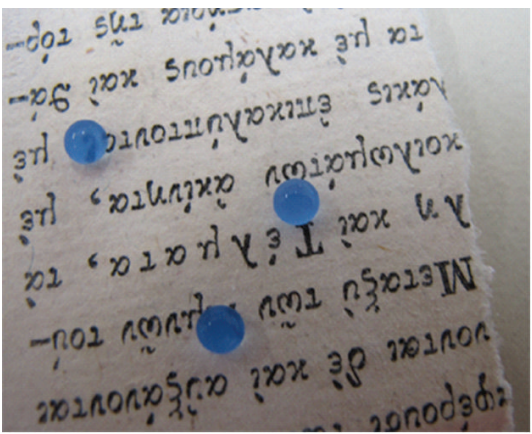

(c)

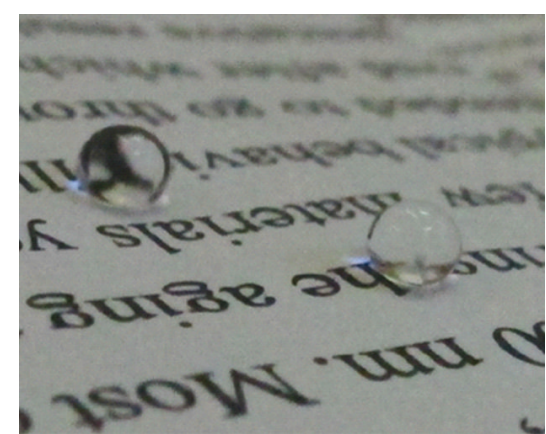

(b)

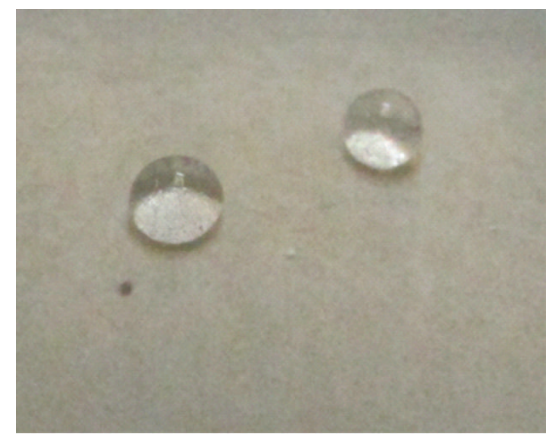

(d)

Figure 5: Photographs of water droplets placed on (a) modern blank, (b) modern printed, (c) old, and (d) Japanese papers, which were covered by siloxane-particle ( $2 \% \mathrm{w} / \mathrm{v})$ films. A blue pigment (cobalt blue) was added in water droplets in (a) and (c). The shape of the droplets is almost spherical indicating the superhydrophobic character of the surfaces of the siloxane-particle films. Furthermore, the photographs demonstrate the transparency of the siloxane-particle films.

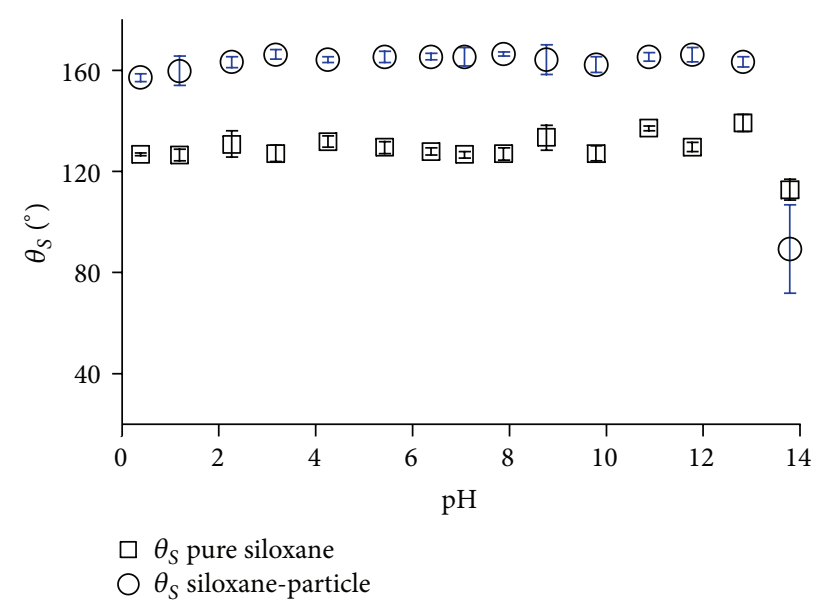

FIGURE 6: Contact angle $\left(\theta_{S}\right)$ versus the $\mathrm{pH}$ of drops on pure siloxane (no particles) and siloxane-particle (2\% w/v) films, deposited on modern blank paper. Error bars are included.

TABLE 2: Colour difference $\left(\Delta E^{*}\right)$ of four paper surfaces, induced by the deposition of pure siloxane and siloxane-particle films, corresponding to 0 and $2 \% \mathrm{w} / \mathrm{v}$ particle concentration, respectively.

\begin{tabular}{lcccc}
\hline Particle concentration (w/v) & \multicolumn{3}{c}{ Paper substrate } & \\
& Modern blank paper & Modern printed paper & Old paper & Japanese paper \\
\hline 0 & $2.86 \pm 0.58$ & $2.44 \pm 0.33$ & $3.24 \pm 0.37$ & $4.34 \pm 0.18$ \\
$2 \%$ & $2.93 \pm 0.29$ & $1.57 \pm 0.17$ & $1.35 \pm 0.34$ & $4.80 \pm 0.21$ \\
\hline
\end{tabular}


(protruded aggregates) which provided the necessary roughness to achieve superhydrophobicity.

The method was applied successfully to treat (i) modern unprinted (blank) paper, (ii) modern paper where a text was printed using a laser jet printer, (iii) a handmade paper sheet detached from an old book (early 20th century), and (iv) Japanese paper used in book conservation and restoration.

Colourimetric measurements showed that the superhydrophobic films had negligible effects on the aesthetic appearance of the treated papers. Furthermore, it was shown that the superhydrophobic character of the siloxane-nanoparticle films is stable over a wide range of $\mathrm{pH}$. The effect of the nanoparticle concentration on the static contact angle $\left(\theta_{S}\right)$ was monitored. It was shown that $\theta_{S}$ measured on surfaces which were prepared from dilute dispersions (particle concentration $<1 \% \mathrm{w} / \mathrm{v}$ ) increased rapidly with particle concentration and reached a maximum value $\left(\sim 162^{\circ}\right)$ at the concentration of around $1 \% \mathrm{w} / \mathrm{v}$. Further increase in particle concentration did not have any effect on $\theta_{S}$.

\section{Conflict of Interests}

The authors declare that there is no conflict of interests regarding the publication of this paper.

\section{Acknowledgment}

Ioannis Karapanagiotis and Katerina E. Aifantis are grateful for the support of the ERC-13 (IL-GradMech-ASM, 88257) program, which is funded by the General Secretariat for Research and Technology (Greece).

\section{References}

[1] J. Song and O. J. Rojas, "Approaching super-hydrophobicity from cellulosic materials: a review," Nordic Pulp \& Paper Research Journal, vol. 28, no. 2, pp. 216-238, 2013.

[2] A. B. D. Cassie and S. Baxter, "Wettability of porous surfaces," Transactions of the Faraday Society, vol. 40, pp. 546-551, 1944.

[3] W. Barthlott and C. Neinhuis, "Purity of the sacred lotus, or escape from contamination in biological surfaces," Planta, vol. 202, no. 1, pp. 1-8, 1997.

[4] J. Shen and X. Qian, "Use of mineral pigments in fabrication of superhydrophobically engineered cellulosic paper," BioResources, vol. 7, no. 4, pp. 4495-4498, 2012.

[5] M. P. Sousa and J. F. Mano, "Patterned superhydrophobic paper for microfluidic devices obtained by writing and printing," Cellulose, vol. 20, no. 5, pp. 2185-2190, 2013.

[6] A. G. Cunha and A. Gandini, "Turning polysaccharides into hydrophobic materials: a critical review. Part 1. Cellulose," Cellulose, vol. 17, no. 5, pp. 875-889, 2010.

[7] D. Nyström, J. Lindqvist, E. Östmark, A. Hult, and E. Malmström, "Superhydrophobic bio-fibre surfaces via tailored grafting architecture," Chemical Communications, no. 34, pp. 35943596, 2006

[8] S. Li, S. Zhang, and X. Wang, "Fabrication of superhydrophobic cellulose-based materials through a solution-immersion process," Langmuir, vol. 24, no. 10, pp. 5585-5590, 2008.
[9] Y.-L. Zhang, J.-N. Wang, Y. He et al., "Solvothermal synthesis of nanoporous polymer chalk for painting superhydrophobic surfaces," Langmuir, vol. 27, no. 20, pp. 12585-12590, 2011.

[10] H. Yang and Y. Deng, "Preparation and physical properties of superhydrophobic papers," Journal of Colloid and Interface Science, vol. 325, no. 2, pp. 588-593, 2008.

[11] M. K. Sarkar, F. He, and J. Fan, "Differential superhydrophobicity and hydrophilicity on a thin cellulose layer," Thin Solid Films, vol. 518, no. 18, pp. 5033-5039, 2010.

[12] C. Quan, O. Werner, L. Wågberg, and C. Turner, "Generation of superhydrophobic paper surfaces by a rapidly expanding supercritical carbon dioxide-alkyl ketene dimer solution," Journal of Supercritical Fluids, vol. 49, no. 1, pp. 117-124, 2009.

[13] H. Wang, J. Fang, T. Cheng et al., "One-step coating of fluorocontaining silica nanoparticles for universal generation of surface superhydrophobicity," Chemical Communications, no. 7, pp. 877-879, 2008.

[14] Z. Hu, X. Zen, J. Gong, and Y. Deng, "Water resistance improvement of paper by superhydrophobic modification with microsized $\mathrm{CaCO}_{3}$ and fatty acid coating," Colloids and Surfaces A: Physicochemical and Engineering Aspects, vol. 351, no. 1-3, pp. 65-70, 2009.

[15] L. Huang, K. Chen, C. Lin, R. Yang, and R. A. Gerhardt, "Fabrication and characterization of superhydrophobic high opacity paper with titanium dioxide nanoparticles," Journal of Materials Science, vol. 46, no. 8, pp. 2600-2605, 2011.

[16] M. Stepien, J. J. Saarinen, H. Teisala et al., "Adjustable wettability of paperboard by liquid flame spray nanoparticle deposition," Applied Surface Science, vol. 257, no. 6, pp. 1911-1917, 2011.

[17] P. Samyn, G. Schoukens, H. van den Abbeele, L. Vonck, and D. Stanssens, "Application of polymer nanoparticle coating for tuning the hydrophobicity of cellulosic substrates," Journal of Coatings Technology Research, vol. 8, no. 3, pp. 363-373, 2011.

[18] D. Stanssens, H. van den Abbeele, L. Vonck, G. Schoukens, M. Deconinck, and P. Samyn, "Creating water-repellent and super-hydrophobic cellulose substrates by deposition of organic nanoparticles," Materials Letters, vol. 65, no. 12, pp. 1781-1784, 2011.

[19] H. Ogihara, J. Xie, J. Okagaki, and T. Saji, "Simple method for preparing superhydrophobic paper: spray-deposited hydrophobic silica nanoparticle coatings exhibit high water-repellency and transparency," Langmuir, vol. 28, no. 10, pp. 4605-4608, 2012.

[20] D. Lampakis, P. N. Manoudis, and I. Karapanagiotis, "Monitoring the polymerization process of Si-based superhydrophobic coatings using Raman spectroscopy," Progress in Organic Coatings, vol. 76, no. 2-3, pp. 488-494, 2013.

[21] P. N. Manoudis, I. Karapanagiotis, A. Tsakalof, I. Zuburtikudis, and C. Panayiotou, "Superhydrophobic composite films produced on various substrates," Langmuir, vol. 24, no. 19, pp. 11225-11232, 2008.

[22] M. K. Tiwari, I. S. Bayer, G. M. Jursich, T. M. Schutzius, and C. M. Megaridis, "Highly liquid-repellent, large-area, nanostructured poly(vinylidene fluoride)/poly(ethyl 2-cyanoacrylate) composite coatings: particle filler effects," ACS Applied Materials \& Interfaces, vol. 2, no. 4, pp. 1114-1119, 2010.

[23] B. J. Basu and V. D. Kumar, "Fabrication of superhydrophobic nanocomposite coatings using polytetrafluoroethylene and silica nanoparticles," ISRN Nanotechnology, vol. 2011, Article ID 803910, 6 pages, 2011.

[24] T. M. Schutzius, I. S. Bayer, G. M. Jursich, A. Das, and C. M. Megaridis, "Superhydrophobic-superhydrophilic binary 
micropatterns by localized thermal treatment of polyhedral oligomeric silsesquioxane (POSS)—silica films," Nanoscale, vol. 4, no. 17, pp. 5378-5385, 2012.

[25] L. Feng, Y. Zhang, J. Xi et al., "Petal effect: a superhydrophobic state with high adhesive force," Langmuir, vol. 24, no. 8, pp. 4114-4119, 2008.

[26] B. Bhushan and M. Nosonovsky, "The rose petal effect and the modes of superhydrophobicity," Philosophical Transactions of the Royal Society A, vol. 368, pp. 4712-4728, 2010.

[27] S. A. Kulinich and M. Farzaneh, "How wetting hysteresis influences ice adhesion strength on superhydrophobic surfaces," Langmuir, vol. 25, no. 16, pp. 8854-8856, 2009.

[28] R. P. S. Chakradhar, V. D. Kumar, C. Shivakumara, J. L. Rao, and B. J. Basu, "Characterisation of microstructure and evaluation of optical and EPR properties of superhydrophobic copper dodecanoate films," Surface and Interface Analysis, vol. 44, no. 4, pp. 412-417, 2012.

[29] M. Nosonovsky and P. K. Rohatgi, Biomimetics in Materials Science. Self-healing, Self-Lubricating, and Self-Cleaning Materials, vol. 152 of Springer Series in Materials Science, 2012.

[30] I. Karapanagiotis, A. Pavlou, P. N. Manoudis, and K. E. Aifantis, "Water repellent ORMOSIL films for the protection of stone and other materials," Materials Letters, vol. 131, pp. 276-279, 2014. 

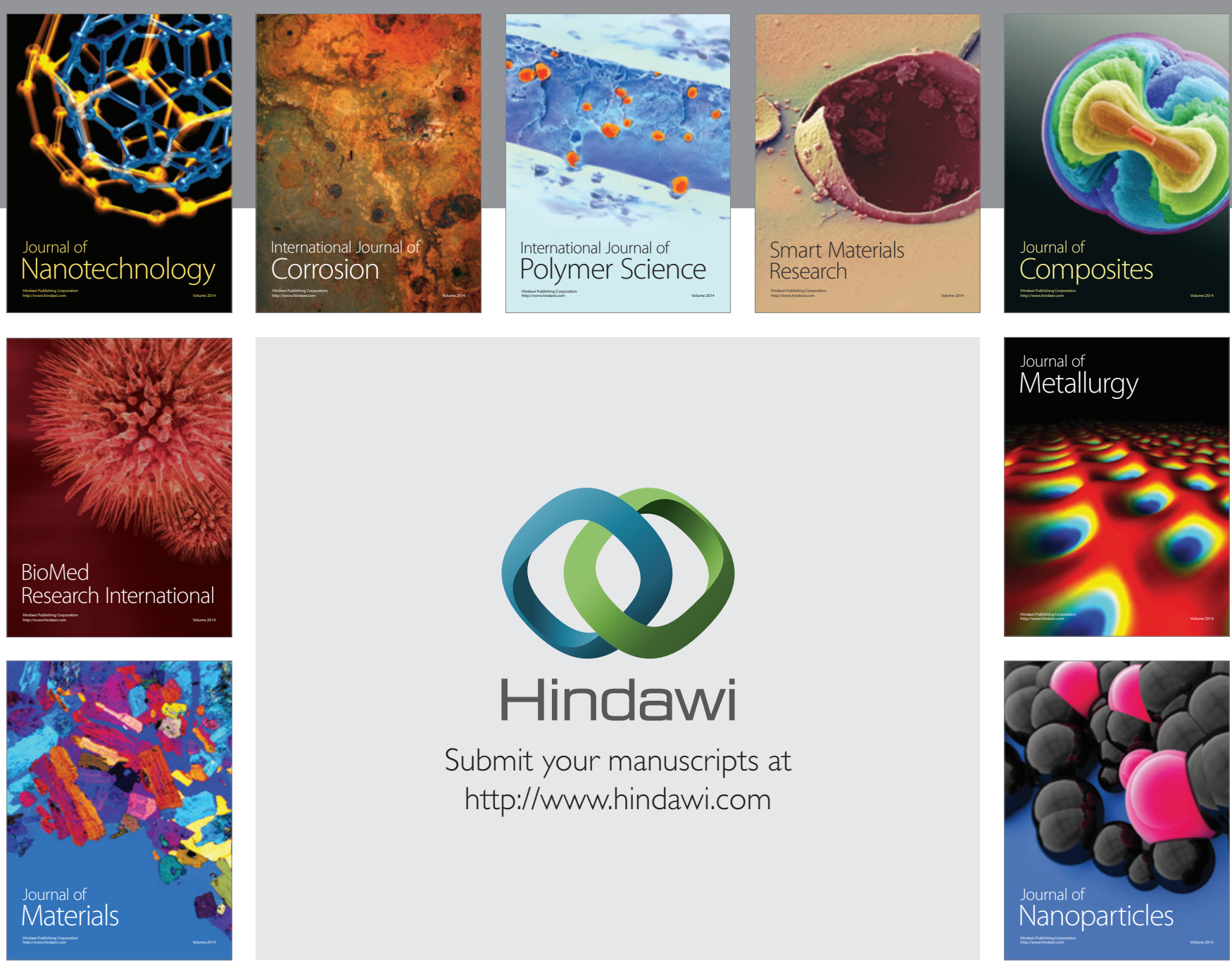

Submit your manuscripts at http://www.hindawi.com
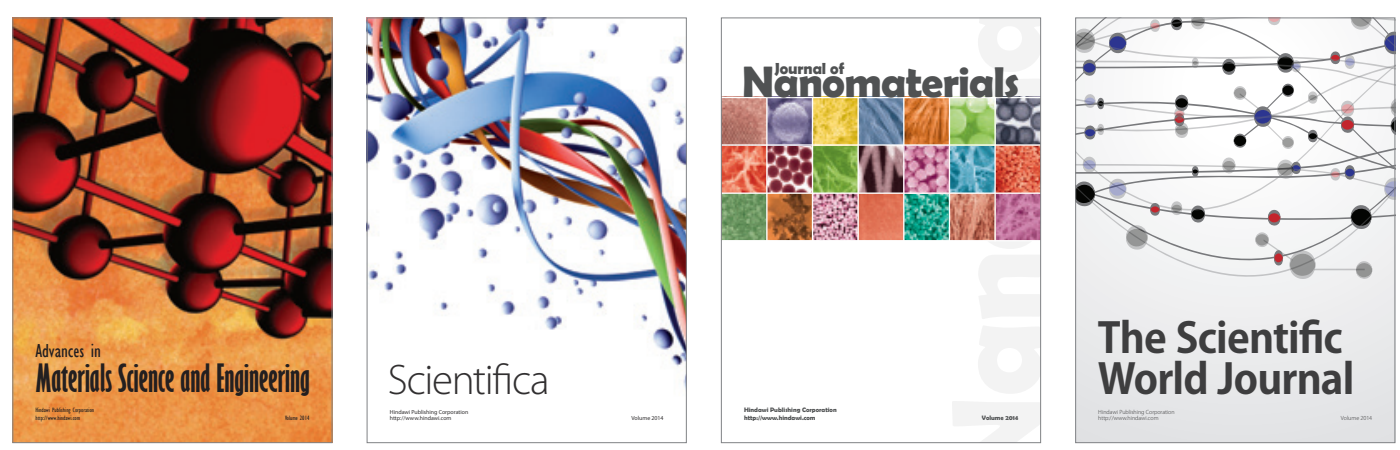

\section{The Scientific World Journal}
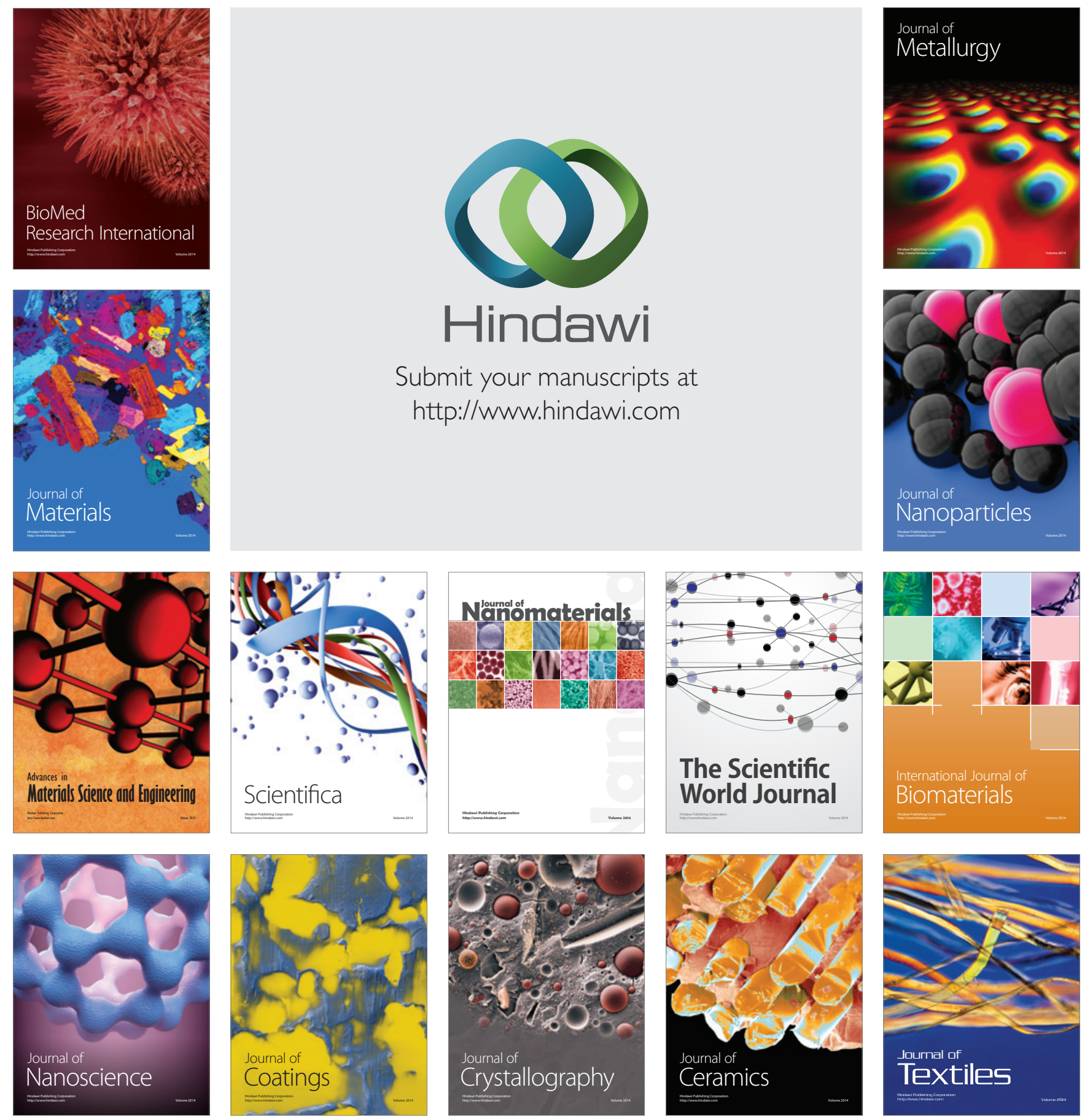